BREAST CANCER

\section{Fibroblast subtypes alter the microenvironment}

Cancer-associated fibroblasts (CAFs) in the tumour microenvironment (TME) have been demonstrated to influence most aspects of tumour biology; however, the effects of fibroblasts expressing different biomarkers can vary dramatically, and this heterogeneity remains poorly understood. Now, the findings of an analysis of tumour and matched nontumour tissue samples from women with breast cancer reveal the existence of four CAF subtypes, with distinct biomarker expression profiles and effects on the TME.

"Many studies have tested different markers for detecting CAFs, most often, each marker is studied individually. However, almost nothing was known about their respective expression pattern in human cancers," lead author Fatima Mechta-Grigoriou explains. The authors conducted an integrated fluorescence-activated cell sorting (FACS) analysis, which enabled the separation of CAFs from other cell types, followed by the quantification of six different fibroblast markers (FAP, integrin $\beta 1, \alpha$-smooth muscle actin, protein S100-A4, PDGFR $\beta$, and caveolin-1) in surgical specimens from women with either luminal A, $\mathrm{HER} 2^{+}$or triple-negative (TNBC) forms of breast cancer.

FACS analysis revealed the existence of four distinct biomarker expression patterns (CAFs-S1-S4), which were subsequently validated in two independent cohorts. Luminal A tumours were found to be enriched predominantly with CAF-S2, HER2 ${ }^{+}$ tumours with CAF-S4, and TNBCs with either CAF-S1 or CAF-S4, although no associations between

CAF subtypes and clinical features were observed in the prospective cohort. Further comparisons of CAF-S1 and CAF-S4 in TNBC samples revealed increased numbers of $\mathrm{CD} 25^{+} \mathrm{CD} 4^{+} \mathrm{T}$ cells, which differentiate into $\mathrm{CD} 25^{+} \mathrm{FOXP} 3^{+} \mathrm{T}$ cells, leading to a reduction in $\mathrm{CD}^{+} \mathrm{T}$ cell abundance, suggesting the emergence of an immunosuppressive TME in patients with TNBCs surrounded by fibroblasts of the CAF-S1 subtype.

In summary, "based on their immunosuppressive activity, CAF-S1 subpopulations could be involved in resistance to immunotherapies" explains Mechta-Grigoriou, adding that "given their key role in tumourigenesis and immunosuppression, targeting the CAF-S1 subset might be a promising therapeutic approach, in combination with the conventional treatments directed against the cancer cells themselves".

Peter Sidaway

ORIGINAL ARTICLE Costa, A. et al. Fibroblast heterogeneity and immunosuppressive environment in human breast cancer. Cancer Cell https://doi.org/10.1016/j.ccell.2018.01.011 (2018) 